\title{
Preface: Ionizing Radiation and Cancer
}

This special issue aims to bring together the leading scholars to provide a cutting-edge overview of the hottest topics in radiobiology and radiation oncology. Although the issue covers a broad spectrum of problems, the bulk is devoted to radiotherapy as an essential part of cancer treatment.

The first part of the issue refers to molecular mechanisms underlying radiation-induced oncogenesis ${ }^{1}$ and radiation-induced cell death. ${ }^{2}$ Recent advances in our understanding of radiation-induced epigenetic alterations, and aberrant DNA methylation in particular, are considered. ${ }^{1}$ The use of DNA methyltransferase inhibitors and methionine dietary interventions targeting the cancer epigenome for radiosensitization seem to be an inherently attractive strategy. The next article summarizes current knowledge on the signaling pathways leading to different modes of regulated cell death after irradiation. ${ }^{2}$ The main principles of regulated cell death modulation as emerging therapeutic means to radiosensitize tumor cells and prevent or mitigate radiation-induced adverse effects are also discussed.

Two subsequent articles deal with an individual variability of the susceptibility to ionizing radiation as the important factor affecting therapeutic outcomes. Chaiswing and co-authors consider in detail the biochemical mechanisms underlying radioadaptive changes and predictive markers of resistance to radiotherapy of prostate cancer. ${ }^{3}$ The novel therapeutic strategies to overcome prostate cancer radioresistance are briefly described. Domina et al. ${ }^{4}$ focus on the early and late responses of normal tissues to therapeutic radiation exposure. The authors discuss how biology-driven personalized radiotherapy could be helpful in identifying patients who are more or less likely to benefit from radiotherapy. Especially useful at this point is the detailed information regarding the role of cancer stem cells in intrinsic tumor radioresistance and treatment outcomes after radiotherapy. The comprehensive table provides the relevant data on candidate molecular markers of tumor radioresponse derived from the most recent and significant clinical studies.
In the second part of the issue, the risks of secondary cancer induced by radiation exposure (both diagnostic and therapeutic) are covered in depth. Balcer-Kubiczek and Eley produce a detailed analysis of the experimental data for relative biological effectiveness for neutrons and different ions used in modern radiotherapy in light of their capacity to induce secondary malignancies among cancer survivors. Much attention is paid to the problems related to the critical assessment of the risks of secondary malignancies by taking into account the detailed description of primary and secondary radiation fields. ${ }^{5}$

Most discussion in the next article ${ }^{6}$ concentrates on the radiation-induced gliomas in terms of their etiology, epidemiology, type of primary tumor, genetic features, radiotherapy modality, and technique. The major risk factors are considered. Finally, the diagnostic and therapeutic uses of radiation as well as the harmful side effects are described with an emphasis on brain tumors. ${ }^{7}$ The understanding of cellular and molecular mechanisms of carcinogenesis in patients treated with radiation will be helpful for decreasing cancer risk after radiation exposure.

We, the editors, deeply thank all authors for their valuable contributions and kind collaborations.

\section{REFERENCES}

1. Miousse IR, Ewing LE, Kutanzi KR, Griffin RJ, Koturbash I. DNA methylation in radiation-induced carcinogenesis: experimental evidence and clinical perspectives. Crit Rev Oncog. 2018;23(1-2):1-11.

2. Philchenkov A. Radiation-induced cell death: signaling and pharmacological modulation. Crit Rev Oncog. 2018;23(1-2):13-37.

3. Chaiswing L, Weiss HL, Jayswal RD, St Clair DK, Kyprianou N. Profiles of radioresistance mechanisms in prostate cancer. Crit Rev Oncog. 2018;23(1-2):39-67.

4. Domina EA, Philchenkov A, Dubrovska A. Individual response to ionizing radiation and personalized radiotherapy. Crit Rev Oncog. 2018;23(1-2):69-92.

5. Balcer-Kubiczek EK, Eley JG. Secondary malignancies in the era of high-precision radiation therapy. Crit Rev Oncog. 2018;23(1-2):93-112.

6. Aherne NJ, Murphy BM. Radiation-induced gliomas. Crit Rev Oncog. 2018;23(1-2):113-18. 
7. Grunert M, Kassubek R, Danz B, Klemenz B, Hasslacher S, Stroh S, Schneele L, Langhans J, Ströbele S, Barry SE, Zhou S, Debatin KM, Westhoff MA. Radiation and brain tumors: an overview. Crit Rev Oncog. 2018;23(1-2):11938 .

\section{Guest Editors:}

Alex Philchenkov, PhD

R.E. Kavetsky Institute of Experimental Pathology, Oncology and Radiobiology

Kyiv, Ukraine

Elizabeth K. Balcer-Kubiczek, PhD

University of Maryland School of Medicine

Marlene and Stewart Greenebaum Cancer Center

Baltimore, MD, USA 